\title{
Letter regarding Xiao WZ et al. entitled "Relationships between PTEN gene mutations and prognosis in glioma: a meta-analysis"
}

\author{
Weichuan Dai ${ }^{1} \cdot$ Junyan Chen $^{1,2} \cdot$ Xieli Guo ${ }^{1} \cdot$ Zhaozhi Su $^{1}$
}

Received: 6 April 2015 / Accepted: 20 April 2015 / Published online: 30 May 2015

(C) International Society of Oncology and BioMarkers (ISOBM) 2015

\begin{abstract}
With great interest, we read the article "Relationships between PTEN gene mutations and prognosis in glioma: a meta-analysis" (by Xiao et al. Tumor Biol 35(7):6687-6693, 2014), which has reached important conclusions that the phosphatase and tensin homolog (PTEN) gene mutations were closely related to poor prognosis of glioma patients. Through quantitative analysis, the investigators (Xiao WZ et al.) showed that glioma patients with PTEN gene mutations exhibited a significantly shorter overall survival (OS) than those without PTEN gene mutations $(\mathrm{HR}=3.66,95 \% \mathrm{CI}=$ 2.02 5.30, $P<0.001)$. Ethnicity-stratified subgroup analysis demonstrated that PTEN gene mutations were closely linked to poor prognosis in glioma among Americans $(\mathrm{HR}=3.72$, $95 \% \mathrm{CI}=1.72 \sim 5.73, P<0.001)$, while similar correlations were not observed among populations in Sweden, Italy, and Malaysia (all $P>0.05$ ). The meta-analysis results are encouraging. Nevertheless, some deficiencies still existed that we would like to raise.
\end{abstract}

Keywords Letter · PTEN gene mutations · Prognosis · Glioma $\cdot$ Meta-analysis

Junyan Chen

drjunyan@126.com

1 Department of Neurosurgery, The Jinjiang City Hospital, Jinjiang 362200, Fujian, China

2 Department of Neurosurgery, The Second Affiliated Hospital, Fujian Medical University, Quanzhou 362000, Fujian, China
Firstly, with respect to the data extraction in the meta analysis [1], two investigators independently extracted data and reached a consensus. We would like to know how to solve the problems, if there are discrepancies between the two investigators.

Secondly, in the results section, the investigators used a forest plot to compare the effect of phosphatase and tensin homolog (PTEN) gene mutation with PTEN gene nonmutation on the overall survival. However, we are wondering what does the overall survival mean, 2-year overall survival or 5 -year overall survival? Meanwhile, the investigators did not evaluate its effect on progression-free survival (PFS). In our opinion, these issues should be added in the results section to make the meta-analysis better.

Finally, the investigators might have made a mistake on the funnel plots. They clarified that "Egger's test also did not illustrate strong statistical evidence of publication bias ( $t=$ $0.12 ; P=0.907)$ " in the results section, while the funnel plot in Fig. 4 is Begg's funnel plot. In our opinion, they must correct this obvious mistake.

Thanks go to the authors for their contribution in supplying us a meta-analysis to explore the associations between PTEN gene mutations and prognosis in glioma. However, further studies with large samples are still needed to study the relationships between PTEN gene mutations and prognosis in glioma.

Conflicts of interest None

\section{Reference}

1. Xiao WZ, Han DH, Wang H, et al. Relationships between PTEN gene mutations and prognosis in glioma: a meta-analysis. Tumor Biol. 2014;35(7):6687-93. 